Research Paper

\title{
Tacrolimus Concentration after Letermovir Initiation in Hematopoietic Stem Cell Transplantation Recipients Receiving Voriconazole: A Retrospective, Observational Study
}

\author{
Shinichi Hikasa ${ }^{\circledR}$, Shota Shimabukuro ${ }^{1}$, Yuko Osugi ${ }^{2}$, Kazuhiro Ikegame $^{2}$, Katsuji Kaida², Keiko \\ Fukunaga ${ }^{2}$, Tomoko Higami ${ }^{1}$, Masami Tada ${ }^{1}$, Kuniyoshi Tanaka ${ }^{1}$, Mina Yanai ${ }^{1}$, and Takeshi Kimura ${ }^{1}$ \\ 1. Department of Pharmacy, Hyogo College of Medicine College Hospital, Nishinomiya, Hyogo 663-8501, Japan \\ 2. Division of Hematology, Department of Internal Medicine, Hyogo College of Medicine, Nishinomiya, Hyogo 663-8501, Japan \\ $\square$ Corresponding author: Shinichi Hikasa, Address: 1-1, Mukogawa-cho, Nishinomiya, Hyogo 663-8501, Japan. Telephone number: 0798-45-6189; Fax number: \\ 0798-45-6190; E-mail address: ykhikasa@hyo-med.ac.jp
}

(c) The author(s). This is an open access article distributed under the terms of the Creative Commons Attribution License (https://creativecommons.org/licenses/by/4.0/). See http://ivyspring.com/terms for full terms and conditions.

Received: 2019.11.12; Accepted: 2020.02.21; Published: 2020.03.15

\begin{abstract}
Letermovir (LMV) is a new antiviral drug used to prevent cytomegalovirus infection in hematopoietic stem cell transplantation (HSCT) recipients. It has been reported to increase tacrolimus (TAC) exposure and decrease voriconazole (VRCZ) exposure in healthy participants. However, VRCZ inhibits the metabolism of TAC. Thus, the effects of LMV on TAC exposure in patients receiving VRCZ are unknown. This retrospective, observational, single-center study was conducted between May 2018 and April 2019. The TAC concentration/dose (C/D) ratio, VRCZ concentration, and VRCZ C/D ratio for 7 days before and for the first and second 7-day periods after the initiation of LMV administration were evaluated. Fourteen HSCT recipients receiving VRCZ were enrolled. There was no significant difference in the TAC $C / D$ ratio for 7 days before and for the first and second 7-day periods after initiating LMV administration (median: 866 [IQR: 653-953], 842 [IQR: 636-1031], and 906 [IQR: 824-1210] [ng/mL]/[mg/kg], respectively). In contrast, the VRCZ C/D ratio and concentration for the first and second 7-day periods after LMV initiation were significantly lower than those before initiating LMV administration (mean $1.11 \pm$ $0.07,0.12 \pm 0.08$, and $0.22 \pm 0.12[\mu \mathrm{g} / \mathrm{mL}] /[\mathrm{mg} / \mathrm{kg}]$ and $0.7 \pm 0.5,0.8 \pm 0.5$, and $1.3 \pm 0.7 \mu \mathrm{g} / \mathrm{mL}$, respectively; $n=12$ ). This can be explained by the increase in TAC concentration caused by CYP3A4 inhibition due to LMV and by the decrease in TAC concentration ascribed to the decrease in VRCZ concentration by CYP2C19 induction due to LMV. These results suggest that it is unnecessary to adjust the dose of TAC based on LMV initiation; however, it is necessary to adjust the dose of TAC based on conventional TAC concentration measurements.
\end{abstract}

Key words: Letermovir, tacrolimus, voriconazole, interaction, hematopoietic stem cell transplantation

\section{Introduction}

Tacrolimus (TAC) has been widely used as an immunosuppressive drug for graft-versus-host disease (GVHD) prophylaxis after hematopoietic stem cell transplantation (HSCT) [1-6]. GVHD remains the major cause of morbidity and mortality in HSCT recipients; therefore, the prevention of severe GVHD is crucial for its successful treatment [7]. Because of the narrow therapeutic range and large intra- and inter-individual variabilities in the pharmacokinetics of TAC [8], adequate blood concentrations of TAC should be maintained to prevent drug-related toxicities and GVHD.

Although evidence regarding voriconazole (VRCZ) prophylaxis in patients receiving HSCT is poor [9], this drug is often used to prevent fungal infections. VRCZ is principally metabolized by 
cytochrome P450 2C19 (CYP2C19) and to a lesser extent by cytochrome P450 3A4 (CYP3A4) and cytochrome P450 2C9 (CYP2C9); it can also be an inhibitor of these enzymes [10-12]. Because TAC is predominantly metabolized by CYP3A4 [13], a significant interaction between VRCZ and TAC, that is, an increase in the whole blood concentration of TAC (TAC concentration) due to the inhibition of CYP3A4 by VRCZ, and the inhibition of TAC metabolism, dependent on the VRCZ concentration, have been reported [12,14-17].

Letermovir (LMV) is a new antiviral drug to prevent cytomegalovirus infection in HSCT recipients [18]. It is a weak-to-moderate inhibitor of CYP3A4 and a weak-to-moderate inducer of CYP2C9 and CYP2C19 [18]. The interaction between LMV and TAC has been assessed in healthy participants, and LMV was found to increase TAC exposure $[19,20]$. The interaction between LMV and VRCZ has also been assessed in healthy participants, and it was found that LMV decreases VRCZ exposure [21]. Therefore, when LMV is administered to patients receiving TAC and $\mathrm{VRCZ}$, a reciprocal effect of LMV on TAC concentration is supposed to occur due to the following: (1) an increase in the TAC concentration due to the inhibition of CYP3A4 by LMV and (2) a decrease in the TAC concentration ascribed to the decrease in VRCZ concentration due to the induction of CYP2C19 by LMV. If (1) is greater than (2), an increase in the TAC concentration is likely to occur, or if (2) is greater than (1), a decrease in the TAC concentration is likely to occur after the initiation of LMV administration. However, it is unknown which is greater, (1) or (2). Specifically, the effects of LMV on TAC in those who receive VRCZ are unknown. Therefore, in this study, we evaluated the effects of LMV on TAC pharmacokinetics by comparing the pharmacokinetic parameters before and after the initiation of LMV administration in HSCT recipients receiving VRCZ. Furthermore, we compared VRCZ pharmacokinetic parameters before and after the initiation of LMV administration to rationalize the participation of VRCZ in TAC pharmacokinetic changes.

\section{Patients and Methods}

\section{Study design and patient population}

This retrospective, observational, single-center study was conducted between May 2018 and April 2019 in Hyogo College of Medicine College Hospital in Japan by using medical chart reviews. Day 0 was defined as the day of the initiation of LMV. The pre-LMV period was defined as 7 days before the initiation of LMV (from day -6 to day 0). The
post-LMV 1 period and post-LMV 2 period were defined as the first and second 7-day periods after the initiation of LMV (from day 1 to day 7 and from day 8 to day 14, respectively). The concentration/dose (C/D) ratio of TAC, plasma VRCZ concentration, and its $\mathrm{C} / \mathrm{D}$ ratio during the pre-LMV period, post-LMV 1 period, and post-LMV 2 period were compared, respectively. The inclusion criteria were as follows: patients for whom LMV administration was initiated between May 2018 and April 2019, patients who received LMV once daily after breakfast (480 $\mathrm{mg} /$ day), patients who received TAC for the prophylaxis of GVHD, and patients who received VRCZ for the prophylaxis of a fungal infection. The following exclusion criteria were applied: patients for whom LMV was discontinued during the post-LMV 1 period or post-LMV 2 period; patients for whom VRCZ was initiated or discontinued during the pre-LMV period, post-LMV 1 period, or post-LMV 2 period; and patients for whom the VRCZ dose was changed during pre-LMV period, post-LMV 1 period, or post-LMV 2 period because TAC concentration is affected by the initiation or discontinuation of VRCZ.

\section{Evaluation of TAC concentration and C/D ratio}

The TAC concentration was measured almost every day. Elecsys TAC assay kits were used to measure the TAC concentration using the Cobas e 801 analytical unit (Roche), according to the manufacturer's protocol. The daily dose of TAC was adjusted based on TAC concentration measurements, and the weight-adjusted dose $(\mathrm{mg} / \mathrm{kg}$ per day) of TAC was calculated. To obtain the C/D ratio of TAC, the measured TAC concentration was divided by the corresponding dose administered $24 \mathrm{~h}$ prior to $6: 00$ a.m. on the day of the TAC concentration measurement per body weight on the day before the TAC concentration measurement. The C/D ratio of TAC during the pre-LMV period, post-LMV 1 period, and post-LMV 2 period was calculated as the mean value for each day during the three periods. The types and doses of proton pump inhibitors were surveyed because proton pump inhibitors increase the TAC concentration [22].

\section{Evaluation of plasma VRCZ concentration and C/D ratio}

The plasma VRCZ concentration (VRCZ concentration) was measured almost weekly. VRCZ was administered orally or intravenously twice daily (10:00 a.m. and 20:00-22:00 p.m.) at a dose that was adjusted based on VRCZ concentration measurements. The VRCZ concentration was determined within $3 \mathrm{~h}$ before the administration of the next dose 
by liquid chromatography-tandem mass spectrometry, which was outsourced to SRL, Inc. (Tokyo, Japan). The VRCZ concentrations during the pre-LMV period, post-LMV 1 period, and post-LMV 2 period were defined as VRCZ concentrations during each of these respective periods. The period from VRCZ initiation to VRCZ concentration measurement was surveyed because it can affect the VRCZ concentration.

\section{Data collection}

The following data at LMV initiation were collected from the electronic medical records: sex, age, height, body weight, disease requiring HSCT, source of stem cells, conditioning regimen, number of HLA mismatches, period from transplantation to LMV initiation, and clinical laboratory parameters (creatinine, total bilirubin, lactate dehydrogenase, aspartate aminotransferase, alanine aminotransferase, alkaline phosphatase, white blood cells, red blood cells, hemoglobin, hematocrit, and platelets).

\section{Statistical methods}

The distribution of continuous variables was determined using the Kolmogorov-Smirnov normality test. Continuous data with a normal distribution and abnormal distribution are shown as mean \pm standard deviation (SD) and median (interquartile range [IQR]) values, respectively. Categorical variables are shown as frequencies and percentages. Based on the results of the KolmogorovSmirnov normality test, repeated-measures analysis of variance (Bonferroni post hoc test) or Friedman tests were used to compare TAC C/D ratios, VRCZ $\mathrm{C} / \mathrm{D}$ ratios, and VRCZ concentrations. The results with a probability value $<0.05$ were considered significant. All analyses were conducted using SPSS statistics version 24.0 software (IBM, Tokyo, Japan).

\section{Ethics statement}

The study protocol was approved by the Ethics Review Board of Hyogo College of Medicine (no. 3186). We obtained consent from all participants included in the study through an opt-out procedure.

\section{Results}

\section{Patient characteristics}

Seventeen patients met the inclusion criteria. Of these, three were excluded based on the exclusion criteria (for one, VRCZ was initiated during the pre-LMV period; for one, the VRCZ dose was changed during the post-LMV 1 period; for one, LMV was discontinued during the post-LMV 1 period), and 14 were enrolled in the study. Table 1 summarizes the clinical characteristics of individuals enrolled in this study and those for whom LMV administration was initiated. All patients received LMV orally. Table 2 summarizes the drugs administered concomitantly with LMV and VRCZ at LMV initiation.

\section{TAC C/D ratio}

There were no significant differences in the C/D ratios of TAC during the pre-LMV period, post-LMV 1 period, and post-LMV 2 period (Table 3). All patients received proton pump inhibitors orally. The types and doses of proton pump inhibitors were the same during the pre-LMV period, post-LMV 1 period, and post-LMV 2 period.

\section{VRCZ C/D ratio and concentration}

Of the 14 patients enrolled in the study, the VRCZ concentration was measured in 12 patients during the pre-LMV period, post-LMV 1 period, and post-LMV 2 period (all patients received oral VRCZ). The mean C/D ratio of VRCZ during the post-LMV 1 period and post-LMV 2 period was significantly lower than that during the pre-LMV period. The mean VRCZ concentration during the post-LMV 1 period and post-LMV 2 period was significantly lower than that during the pre-LMV period (Table 3). In two, six, three, and one patient(s), the VRCZ concentration during the pre-LMV period was measured on day -4 , $-3,-1$, and 0 , respectively. In two, five, four, and one patient(s), the VRCZ concentration during the post-LMV 1 period was measured on day $3,4,6$, and 7 , respectively. In two, six, three, and one patient(s), the VRCZ concentration during the post-LMV 2 period was measured on day 10,11, 13, and 14, respectively. The period from VRCZ initiation to VRCZ measurement during the pre-LMV period was more than 14 days for all patients.

\section{Discussion}

To the best of our knowledge, this is the first study to assess the effect of LMV on TAC in HSCT recipients receiving VRCZ. The results demonstrated that there was no significant difference in the $C / D$ ratios of TAC before and after LMV initiation. This can be explained by the increase in the TAC concentration by CYP3A4 inhibition due to LMV and by the decrease in TAC concentration by CYP2C19 induction due to LMV.

It is important to evaluate the interaction at the time when the enzyme is sufficiently inhibited or induced. It is generally assumed that enzyme inducers accelerate enzyme synthesis in a concentration-dependent manner. Since enzyme synthesis is assumed to obey zero-order kinetics, the effect of the inducer on enzyme synthesis starts immediately. Therefore, the gradual increase in CYP 
activity over several days of exposure to the inducer is attributed simply to the slow degradation rate of these enzymes [23]. Stable trough levels of LMV were reached on day 4 after LMV initiation [24]. However, several studies have indicated that it takes at least 24 to $72 \mathrm{~h}$ for the CYP activity of the enzyme inducer to reach a maximum level $[25,26]$. It is unclear when CYP2C19 induction due to LMV reaches a maximum level. However, in a pharmacokinetic trace of LMV co-administered with VRCZ, the pharmacokinetics were assessed on day 8 after the 7-day administration of LMV [21]. Therefore, in the present study, the interaction was evaluated during the pre-LMV period, post-LMV 1 period, and post-LMV 2 period (each for 7 days).

Table 1. Patient and transplantation characteristics

\begin{tabular}{|c|c|}
\hline Patients, $n$ & 14 \\
\hline Men, $n(\%)$ & $11(79)$ \\
\hline Age, years & $44 \pm 11$ \\
\hline Height, cm & $172(167,176)$ \\
\hline Body weight, kg & $62.9 \pm 8.6$ \\
\hline \multicolumn{2}{|l|}{ Disease } \\
\hline Acute myeloid leukemia, $n(\%)$ & $5(36)$ \\
\hline Acute lymphocytic leukemia, $n(\%)$ & $4(29)$ \\
\hline Myelodysplastic syndromes, $n(\%)$ & $2(14)$ \\
\hline Lymphoblastic lymphoma, $n(\%)$ & $2(14)$ \\
\hline Diffuse large B-cell lymphoma, $n(\%)$ & $1(7)$ \\
\hline \multicolumn{2}{|l|}{ Source of stem cells } \\
\hline Peripheral blood, $n(\%)$ & $13(93)$ \\
\hline Bone marrow, $n(\%)$ & $1(7)$ \\
\hline \multicolumn{2}{|l|}{ Conditioning regimen } \\
\hline Myeloablative, $n(\%)$ & $1(7)$ \\
\hline Reduced intensity, $n(\%)$ & $13(93)$ \\
\hline \multicolumn{2}{|l|}{ Number of HLA mismatches } \\
\hline $1, n(\%)$ & $1(7)$ \\
\hline $2, n(\%)$ & $0(0)$ \\
\hline$\geq 3, \mathrm{n}(\%)$ & $13(93)$ \\
\hline Period from transplantation to LMV initiation, days & $3(3,4)$ \\
\hline Creatinine, $\mathrm{mg} / \mathrm{dL}$ & $0.58(0.41,0.86)$ \\
\hline Total bilirubin, $\mathrm{mg} / \mathrm{dL}$ & $0.4(0.3,0.9)$ \\
\hline Lactate dehydrogenase, IU/L & $235(169,292)$ \\
\hline Aspartate aminotransferase, IU/L & $19 \pm 9$ \\
\hline Alanine aminotransferase, median, IU/L & $21(14,32)$ \\
\hline Alkaline phosphatase, IU/L & $255 \pm 65$ \\
\hline White blood cell, $/ \mu \mathrm{L}$ & $165(50,300)$ \\
\hline Red blood cell, $\times 10^{4} / \mu \mathrm{L}$ & $289(270,299)$ \\
\hline Hemoglobin, $\mathrm{g} / \mathrm{dL}$ & $8.8 \pm 0.8$ \\
\hline Hematocrit, \% & $25.2 \pm 2.4$ \\
\hline Platelet, $\times 10^{4} / \mu \mathrm{L}$ & $3.5(2.7,4.8)$ \\
\hline \multicolumn{2}{|l|}{ Route of voriconazole administration } \\
\hline Oral administration, $n(\%)$ & $13(93)$ \\
\hline Drip infusion, $n(\%)$ & $1(7)$ \\
\hline
\end{tabular}

Table 2. Drugs administered concomitantly with LMV and VRCZ at LMV initiation

Antiviral agent

Acyclovir, $n(\%)$

Antimicrobial agent

Moxifloxacin hydrochloride, $n(\%)$
Meropenem, $n(\%)$

$12(86)$

Tazobactam/piperacillin, $n(\%)$

$2(14)$

Linezolid, $n(\%)$

Antifungal agent

Caspofungin, $n(\%)$

Proton pump inhibitor

Lansoprazole, $n(\%)$

Esomeprazole, $n$ (\%)

Corticosteroid

Methylprednisolone, $n$ (\%)

Prednisolone, $n(\%)$

Other

Ursodeoxycholic acid, $n(\%)$

Lenograstim, $n(\%)$

Danaparoid sodium, $n(\%)$

Amlodipine, $n(\%)$

Brotizolam, $n(\%)$

Zolpidem, $n(\%)$

Furosemide, $n(\%)$

6 (43)

8 (57)

11 (79)

2 (14)

11 (79)

2 (14)

14 (100)

10 (71)

9 (64)

3 (21)

2 (14)

2 (14)

2 (14)

Data do not include infusions. Each one patient received atovaquone, pregabalin, alendronate, polaprezinc, L-carbocisteine, fexofenadine, magnesium oxide,

febuxostat, sitagliptin, rabeprazole, levofloxacin, Lactobacillus preparation,

daptomycin, aztreonam, metoclopramide, defibrotide, carperitide, teicoplanin,

panthenol, and liposomal amphotericin B.

Table 3. TAC C/D ratio, VRCZ C/D ratio, and VRCZ concentration before and after LMV initiation

\begin{tabular}{lllll}
\hline & $\begin{array}{l}\text { Pre-LMV } \\
\text { period }\end{array}$ & $\begin{array}{l}\text { Post-LMV 1 } \\
\text { period }\end{array}$ & $\begin{array}{l}\text { Post-LMV 2 } \\
\text { period }\end{array}$ & $\begin{array}{l}\text { p } \\
\text { value }\end{array}$ \\
\hline TAC C/D ratio, $(\mathbf{n g} / \mathbf{m L}) /(\mathbf{m g} / \mathbf{k g})$ & $866(653$, & $842(636$, & $906(824$, & 0.931 \\
& $953)$ & $1031)$ & $1210)$ & \\
VRCZ C/D ratio, & $0.22 \pm 0.12$ & $0.11 \pm 0.07$ & $0.12 \pm 0.08$ & 0.005 \\
$(\mu \mathrm{gg} / \mathrm{mL}) /(\mathbf{m g} / \mathbf{k g})$ & & 0.029 & 0.007 & \\
p value (vs pre-LMV period) & & & 1.000 & \\
p value (vs post-LMV 1 period) & & $0.7 \pm 0.5$ & $0.8 \pm 0.5$ & 0.003 \\
VRCZ concentration, $\mu \mathrm{g} / \mathrm{mL}$ & $1.3 \pm 0.7$ & 0.023 & 0.006 & \\
p value (vs pre-LMV period) & & & 1.000 & \\
p value (vs post-LMV 1 period) & & & & \\
\hline LMV: letermovir; VRCZ: voriconazole; C/D: concentration/dose &
\end{tabular}

In a previous study, the co-administration of LMV at clinical doses with TAC resulted in a 2.4-fold increase in the area under the plasma TAC concentration-time curve and a 1.6-fold increase in the maximum plasma TAC concentration in 14 healthy female participants [20]. This was because TAC is predominantly metabolized by CYP3A4 [13] and LMV is a moderate inhibitor of CYP3A4 [18]. In contrast, the effect of LMV on VRCZ was examined in 14 healthy subjects [21]. In that study, the area under the curve and peak concentration geometric mean ratios for $\mathrm{VRCZ}+\mathrm{LMV} / \mathrm{VRCZ}$ alone were 0.56 and 0.61 , respectively. This might be because VRCZ is eliminated largely via hepatic metabolism by CYP2C19, with a minor contribution from CYP3A4 and CYP2C9 [10,11], and LMV induces weak-to-moderate CYP2C19 and CYP2C9 activities [18]. Moreover, the inhibition of TAC metabolism is positively dependent on the VRCZ concentration due to the inhibition of CYP3A4 by VRCZ [15-17]. Guo et al. reported three cases in which TAC and LMV were administered [27]. In their study, two cases without VRCZ administration showed a significant increase in 
the TAC concentration; however, the TAC concentration did not fluctuate greatly in one case with VRCZ administration. The findings in these cases seemed to support our results.

Regarding VRCZ, an analysis of the exposureresponse relationship indicated that the optimal target plasma trough concentrations ranged between 1.5 and $4.5 \mu \mathrm{g} / \mathrm{mL}$ [28] and therapeutic drug monitoring is recommended to maximize therapeutic success [29]. However, the present study showed a considerable decrease in VRCZ concentrations after LMV initiation, and the concentration decreased to levels lower than optimal target plasma trough concentrations. Thus, frequent measurements of VRCZ concentrations might be necessary to maintain the optimal target plasma trough concentration range when LMV is initiated in patients receiving VRCZ.

Our study has several limitations. First, the present study had a retrospective observation design without a control group and not as an intervention trial. Thus, the magnitude of interactions was not considered. Second, in this study, polymorphisms in genes encoding metabolic enzymes (CYP3A4, CYP3A5, CYP2C9, and CYP2C19) were not assessed. In one participant, the $\mathrm{C} / \mathrm{D}$ ratios of TAC were $>2000$ $(\mathrm{ng} / \mathrm{mL}) /(\mathrm{mg} / \mathrm{kg})$. This might be affected by gene polymorphisms. Further studies are required to address this.

\section{Conclusion}

The present study demonstrated that the C/D ratio of TAC does not significantly change and that the VRCZ concentration significantly decreases after the initiation of LMV administration.

\section{Abbreviations}

C/D: concentration/dose; CYP: cytochrome P450; GVHD: graft-versus-host disease; HSCT: hematopoietic stem cell transplantation; IQR: interquartile range; LMV: letermovir; SD: standard deviation; TAC: tacrolimus; VRCZ: voriconazole.

\section{Acknowledgments}

Funding: This work was supported by the Japan Society for the Promotion of Science KAKENHI (Grant number JP19H00364).

\section{Competing Interests}

The authors have declared that no competing interest exists.

\section{References}

1. Ratanatharathorn V, Nash RA, Przepiorka D, et al. Phase III study comparing methotrexate and tacrolimus (prograf, FK506) with methotrexate and cyclosporine for graft-versus-host disease prophylaxis after HLA-identical sibling bone marrow transplantation. Blood. 1998; 92: 2303-14.
2. Przepiorka D, Khouri I, Ippoliti C, et al. Tacrolimus and minidose methotrexate for prevention of acute graft-versus-host disease after HLA-mismatched marrow or blood stem cell transplantation. Bone Marrow Transplant. 1999; 24: 763-8.

3. Nash RA, Antin JH, Karanes C, et al. Phase 3 study comparing methotrexate and tacrolimus with methotrexate and cyclosporine for prophylaxis of acute graft-versus-host disease after marrow transplantation from unrelated donors. Blood. 2000; 96: 2062-8.

4. Hiraoka A, Ohashi Y, Okamoto S, et al. Phase III study comparing tacrolimus (FK506) with cyclosporine for graft-versus-host disease prophylaxis after allogeneic bone marrow transplantation. Bone Marrow Transplant. 2001; 28: $181-5$.

5. Yanada M, Emi N, Naoe T, et al. Tacrolimus instead of cyclosporine used for prophylaxis against graft-versus-host disease improves outcome after hematopoietic stem cell transplantation from unrelated donors, but not from HLA-identical sibling donors: a nationwide survey conducted in Japan. Bone Marrow Transplant. 2004; 34: 331-7.

6. Murata M. Prophylactic and therapeutic treatment of graft-versus-host disease in Japan. Int J Hematol. 2015; 101: 467-86.

7. Gooley TA, Chien JW, Pergam SA, et al. Reduced mortality after allogeneic hematopoietic-cell transplantation. N Engl J Med. 2010; 363: 2091-101.

8. Venkataramanan R, Swaminathan A, Prasad T, et al. Clinical pharmacokinetics of tacrolimus. Clin Pharmacokinet. 1995; 29: 404-30.

9. Mellinghoff SC, Panse J, Alakel N, et al. Primary prophylaxis of invasive fungal infections in patients with haematological malignancies: 2017 update of the recommendations of the Infectious Diseases Working Party (AGIHO) of the German Society for Haematology and Medical Oncology (DGHO). Ann Hematol. 2018; 97: 197-207.

10. Theuretzbacher U, Ihle F, Derendorf H. Pharmacokinetic/pharmacodynamic profile of voriconazole. Clin Pharmacokinet. 2006; 45: 649-63.

11. Eiden C, Cociglio M, Hillaire-Buys D, et al. Pharmacokinetic variability of voriconazole and $\mathrm{N}$-oxide voriconazole measured as therapeutic drug monitoring. Xenobiotica. 2010; 40: 701-6.

12. Saad AH, DePestel DD, Carver PL. Factors influencing the magnitude and clinical significance of drug interactions between azole antifungals and select immunosuppressants. Pharmacotherapy. 2006; 26: 1730-44.

13. Sattler M, Guengerich FP, Yun $\mathrm{CH}$, et al. Cytochrome P-450 3A enzymes are responsible for biotransformation of FK506 and rapamycin in man and rat. Drug Metab Dispos. 1992; 20: 753-61.

14. Dodds-Ashley E. Management of drug and food interactions with azole antifungal agents in transplant recipients. Pharmacotherapy. 2010; 30: 842-54.

15. Imamura CK, Furihata K, Okamoto S, et al. Impact of cytochrome P450 2C19 polymorphisms on the pharmacokinetics of tacrolimus when coadministered with voriconazole. J Clin Pharmacol. 2016; 56: 408-13.

16. Mochizuki E, Furuhashi $\mathrm{K}$, Fujisawa $\mathrm{T}$, et al. A case of treatment with voriconazole for chronic progressive pulmonary aspergillosis in a patient receiving tacrolimus for dermatomyositis-associated interstitial lung disease. Respir Med Case Rep. 2015; 16: 163-5.

17. Venkataramanan R, Zang S, Gayowski T, et al. Voriconazole inhibition of the metabolism of tacrolimus in a liver transplant recipient and in human liver microsomes. Antimicrob Agents Chemother. 2002; 46: 3091-3.

18. Marty FM, Ljungman $\mathrm{P}$, Chemaly RF, et al. Letermovir prophylaxis for cytomegalovirus in hematopoietic-cell transplantation. N Engl J Med. 2017; 377: 2433-44.

19. Kropeit D, von Richter O, Stobernack HP, et al. Pharmacokinetics and safety of letermovir coadministered with cyclosporine A or tacrolimus in healthy subjects. Clin Pharmacol Drug Dev. 2018; 7: 9-21.

20. McCrea JB, Macha S, Adedoyin A, et al. Pharmacokinetic drug-drug interactions between letermovir and the immunosuppressants cyclosporine, tacrolimus, sirolimus, and mycophenolate mofetil. J Clin Pharmacol. 2019; 59: 1331-9.

21. Marshall WL, McCrea JB, Macha S, et al. Pharmacokinetics and tolerability of letermovir coadministered with azole antifungals (posaconazole or voriconazole) in healthy subjects. J Clin Pharmacol. 2018; 58: 897-904.

22. Fu R, Tajima $S$, Suetsugu $K$, et al. Biomarkers for individualized dosage adjustments in immunosuppressive therapy using calcineurin inhibitors after organ transplantation. Acta Pharmacol Sin. 2019; 40: 151-9.

23. Yamashita F, Sasa Y, Yoshida S, et al. Modeling of rifampicin-induced CYP3A4 activation dynamics for the prediction of clinical drug-drug interactions from in vitro data. PLoS One. 2013; 8: e70330.

24. Stoelben S, Arns W, Renders L, et al. Preemptive treatment of Cytomegalovirus infection in kidney transplant recipients with letermovir: results of a Phase 2a study. Transpl Int. 2014; 27: 77-86.

25. Pascussi JM, Robert A, Nguyen M, et al. Possible involvement of pregnane $X$ receptor-enhanced CYP24 expression in drug-induced osteomalacia. J Clin Invest. 2005; 115: 177-86.

26. Zhang JG, Ho T, Callendrello AL, et al. A multi-endpoint evaluation of cytochrome P450 1A2, 2B6 and 3A4 induction response in human hepatocyte cultures after treatment with beta-naphthoflavone, phenobarbital and rifampicin. Drug Metab Lett. 2010; 4: 185-94.

27. Guo YM, Abumiya M, Yamashita T, et al. Pharmacokinetics of tacrolimus coadministered with letermovir in allogeneic hematopoietic stem cell transplantation patients. Clin Pharmacol Drug Dev. 2019; 8: 411-2.

28. Pascual A, Csajka C, Buclin T, et al. Challenging recommended oral and intravenous voriconazole doses for improved efficacy and safety: population 
pharmacokinetics-based analysis of adult patients with invasive fungal infections. Clin Infect Dis. 2012; 55: 381-90.

29. Brüggemann RJM, Donnelly JP, Aarnoutse RE, et al. Therapeutic drug monitoring of voriconazole. Ther Drug Monit. 2008; 30: 403-11.

\section{Author Biography}

Shinichi Hikasa is a Sub Chief Pharmacist in the Department of Pharmacy at the Hospital of Hyogo College of Medicine. He is the author of eight original papers published in international and national journals. His main interests are in the field of stewardship of antiviral drug use. 